\title{
Brain Tumor Detection and Segmentation Using Watershed Segmentation and Morphological Operation
}

\author{
V. Supraja ${ }^{1}$, Kuna Haritha ${ }^{2}$, Gunjalli Mounika ${ }^{2}$, Chintha Manideepika ${ }^{2}$, Kandikeri Sai Jeevani ${ }^{2}$ \\ ${ }^{1}$ Associate Professor, Department of Electronics \& Communication Engineering, Ravindra College of \\ Engineering for Women, Kurnool, India \\ ${ }^{2}$ Student, Department of Electronics \& Communication Engineering, Ravindra College of Engineering for \\ Women, Kurnool, India
}

\begin{abstract}
Article Info

Volume 8, Issue 4

Page Number : 304-312

Publication Issue :

July-August-2021

Article History

Accepted : 01 Aug 2021

Published: 07 Aug 2021

In the field of medical image processing, detection of brain tumor from magnetic resonance image (MRI) brain scan has become one of the most active research. Detection of the tumor is the main objective of the system. Detection plays a critical role in biomedical imaging. In this paper, MRI brain image is used to tumor detection process. This system includes test the brain image process, image filtering, skull stripping, segmentation, morphological operation, calculation of the tumor area and determination of the tumor location. In this system, morphological operation of erosion algorithm is applied to detect the tumor. The detailed procedures are implemented using MATLAB. The proposed method extracts the tumor region accurately from the MRI brain image. The experimental results indicate that the proposed method efficiently detected the tumor region from the brain image. And then, the equation of the tumor region in this system is effectively applied in any shape of the tumor region.

Keywords : Magnetic resonance image, skull stripping, segmentation, morphological operation, detection
\end{abstract}

\section{INTRODUCTION}

In the medical field, magnetic resonance image (MRI) is widely used in many research. MRI techniques is a noninvasive method and uses powerful magnet and radio waves to create the picture of the body. It is suited for examining soft tissues of the human body such as Ligament and tendon injury, spinal cord injury and brain tumors, etc. The detail information of the human brain can be get using MRI techniques.
Brain, heart and lung etc. are the most important parts of the human body. And then, all parts of the body are controlled by the brain cells. Therefore, brain is a vital organ of the body. Nowadays, brain tumor is a very serious disease among children and adults. The most deadly and intractable diseases are brain tumor. Brain tumor's location and quickly spreading make a critical problem in treatment of tumor. Thus, image segmentation and detection are 
vital method to solve the medical problem of the various diseases. Imaging of the brain tumor can be done by computer tomography (CT) scan, magnetic resonance image (MRI) scan, Ultrasound, etc. In this research, MRI scan is used to implement the system.

Several works for detection of the brain tumor have been reported. Pratik P et al proposed brain tumor detection method using connected component analysis. The method proposed by Manor K Kowari et al requires to do image cropping so that the exact result of the tumor region is not obtained. M. Masroor Ahmed et al proposed the method of the brain tumor detection using Kmeans Clustering. Nagalkar V J et al proposed brain tumor detection using soft computing method. This method can cause false detection in seeing scan. Rajesh C. Patil et al proposed the method of the brain tumor extraction from MRI images using MATLAB. Image segmentation can be done by various techniques: histogram thresholding, region growing, K-means Clustering and watershed segmentation Watershed segmentation is suitable for tumor region that have higher intensity values. In this paper, marker-controlled watershed segmentation is used to prevent over segmentation. Preprocessing of the MRI image is the primary step which removes noise and smooth the image. To prevent misclassification of brain tissue and non-brain tissues, skull stripping is done. And, image segmentation is carried out using marker-controlled watershed segmentation. Then, the tumor region is detected from the segmented image using morphological operation and calculated the tumor region. Finally, the location of the tumor region is determined.

\section{MATERIALS AND METHODS}

\subsection{Pre-processing}

Preprocessing include the input MRI brain tumor image and image filtering. In image filtering, several different filters can be used but the magnetic resonance image (MRI) image does not contain a lot of noise. So, in this research, average filter is used to smooth the image. The smoothed image is used to operate the next step of the system quickly. Average filter is low pass filter. Average filter is a simple and easy to implement method of smoothing images. The operation of average filter is

$$
\mathrm{g}(\mathrm{x}, \mathrm{y})=1 / \mathrm{M} \sum_{(x, y) \in s} f(x, y)
$$

Where, S=neighborhood of pixel $(x, y)$

$\mathrm{M}=$ number of pixels in neighborhood $\mathrm{S}$

\subsection{Skull Stripping}

Skull stripping is important process in biomedical image analysis. It is needed to make only in brain image but is not needed to make in other medical image analysis such as heart, lung, etc. It must be done before other image processing step. It is a process of eliminating all non-brain tissues from brain image. In skull stripping, it is removed extra cerebral tissues such as skull, fat, skin, etc. Skull stripping can be done by various methods. They are automatic skull stripping using image contour, skull stripping based on region growing and mathematical morphology, skull stripping based on histogram analysis, skull stripping based on resonance principle and skull stripping based on threshold value. Skull stripping based on threshold value is used to remove the skull tissues in this paper. In the skull stripping based on threshold value, the threshold value of the skull tissues and that of normal brain tissues are manually determined for every image [13].

\subsection{Watershed Segmentation}

Watershed segmentation is a gradient-based segmentation technique. It considers the gradient map of the image as a relief map. It segments the image as a dam. The segmented regions are called catchment basins. Watershed segmentation solves a variety of image segmentation problem. It is suitable for the images that have higher intensity value. Watershed segmentation is caused over segmentation. 
To control over segmentation, marker controlled watershed segmentation is used. Sobel operator is suitable for edge detection. In marker controlled watershed segmentation, sobel operator is used to distinct the edge of the object [10].

The sobel masks in matrix form are as follow

$M_{X}=\left[\begin{array}{rrr}-1 & -2 & -1 \\ 0 & 0 & 0 \\ 1 & 2 & 1\end{array}\right], M_{y}=\left[\begin{array}{lll}-1 & 0 & 1 \\ -2 & 0 & 0 \\ -1 & 0 & 2\end{array}\right]$

The equation of gradient magnitude used in marker controlled watershed segmentation is

Angle, $\theta^{=} \tan ^{-1} \frac{M y}{M x}$

\subsection{Morphological Operation}

Morphological image processing is a collection of non- linear operations related to the shape or morphology of features in an image. A morphological operation on a binary image creates a new binary image in which the pixel has a non-zero value. Morphological operations transform the image. In this paper, erosion is applied to detect the tumor [6]. The erosion of $\mathrm{A}$ by $\mathrm{B}$ is given by the expression:

$\mathrm{A} \Theta \mathrm{B}=\{(\mathrm{i}, \mathrm{j}): \mathrm{B}(\mathrm{i}, \mathrm{j})\} \quad \mathrm{CA}$

Where, $A=$ the binary image,

$\mathrm{B}=$ the structuring element

$(i, j)=$ the center pixel of structuring element

\subsection{Calculation the tumor region}

The area of the tumor region is calculated by the following equation:

Tumor area $=$ Axtotal number of pixel in the tumor region (5) $\mathrm{A}=\mathrm{V} \times \mathrm{H}$

Where, $A=$ the area of each pixel

$\mathrm{H}=$ horizontal dimension of the image $\mathrm{V}=$ vertical dimension of the image $\mathrm{H}=1$ /horizontal resolution of the image $\mathrm{V}=1$ /vertical resolution of the image.

\section{IMPLEMENTATION}

In this paper, the system is implemented as shown in Fig-1. In this system, the input MRI brain image is used to implement the algorithms. MRI image gives the detailed information of the brain tissues than other scan of the brain. Firstly, the brain image is checked out as the brain image with tumor region or the brain image without tumor region. So, the tumor region is extracted from the brain image and the number of pixel for the tumor region is calculated. If the number of pixel for the tumor region is equal to zero, the system displays the brain image without tumor region.

Otherwise, the system displays the brain image with tumor region. If the brain image has tumor region, this image is needed to do the following further steps. In the next step, the noise from the brain image is removed using filter. Several filters can be used to remove the noise. But, the MRI image does not contain a lot of noise. So the average filter is used to remove the noise from the brain image in this system. After preprocessing, the smoothed image is obtained and it is ready to use in the next step. The smoothed image is used to operate the next step quickly. In brain image, skull stripping is needed to do for avoiding misclassification. Skull stripping can be done by various method. In this paper, skull stripping based on threshold value is used to extract the skull tissues. In process, the brain tissues are firstly extracted from the brain image and the holes are filled the brain image. And then, the skull tissues are extracted from the filled image and are given the name, the skull image. The skull image is converted into the binary image. The binary image and the inverse of the image that extract brain tissues image are combined to get the skull border. When the skull border in the brain image is given zero, the brain image without skull tissues is get. After the brain image without skull tissues had been obtained, segmentation process is done. In segmentation step, the brain image obtained 
from the skull stripping step is segmented using marker-controlled watershed segmentation.

This segmentation process uses the sobel operator to find the gradient image. Using the gradient image, the watershed transform is estimated. Next, the segmented image is divided into normal brain region and tumor region according to intensity value of the original image. Therefore, the final segmentation map is get. Then, the tumor region is detected from the final segmentation map using morphological operation. In this process, the image get from the segmentation step is eroded. And then, the gray matter and white matter are extracted from the brain image. The region properties of the image are defined and the values of the regions are calculated. And the region that has the maximum intensity value is found out. Finally, the tumor region is efficiently detected from the brain image. After the tumor region had been detected from the brain image, the area of the tumor region is calculated. Firstly, the area of the pixel is calculated. Then, the total pixel of the tumor region is calculated. And then, the area of the tumor region is calculated. Next, the location of the tumor region is determined. The tumor image obtained from the detection process is divided into two parts. The right part of the image is defined as the left hemisphere of the brain and the left part of the image is the right hemisphere of the brain. Then the total number of pixel for each part is calculated and compared them. If the total number of pixel of each part is equal, the tumor is located in the center of the brain. Otherwise, the other two conditions are checked out. If the pixel value of the right part of the image is greater than that of the left part, the tumor is located in the left hemisphere of the brain and else the tumor is located in the right hemisphere of the brain.

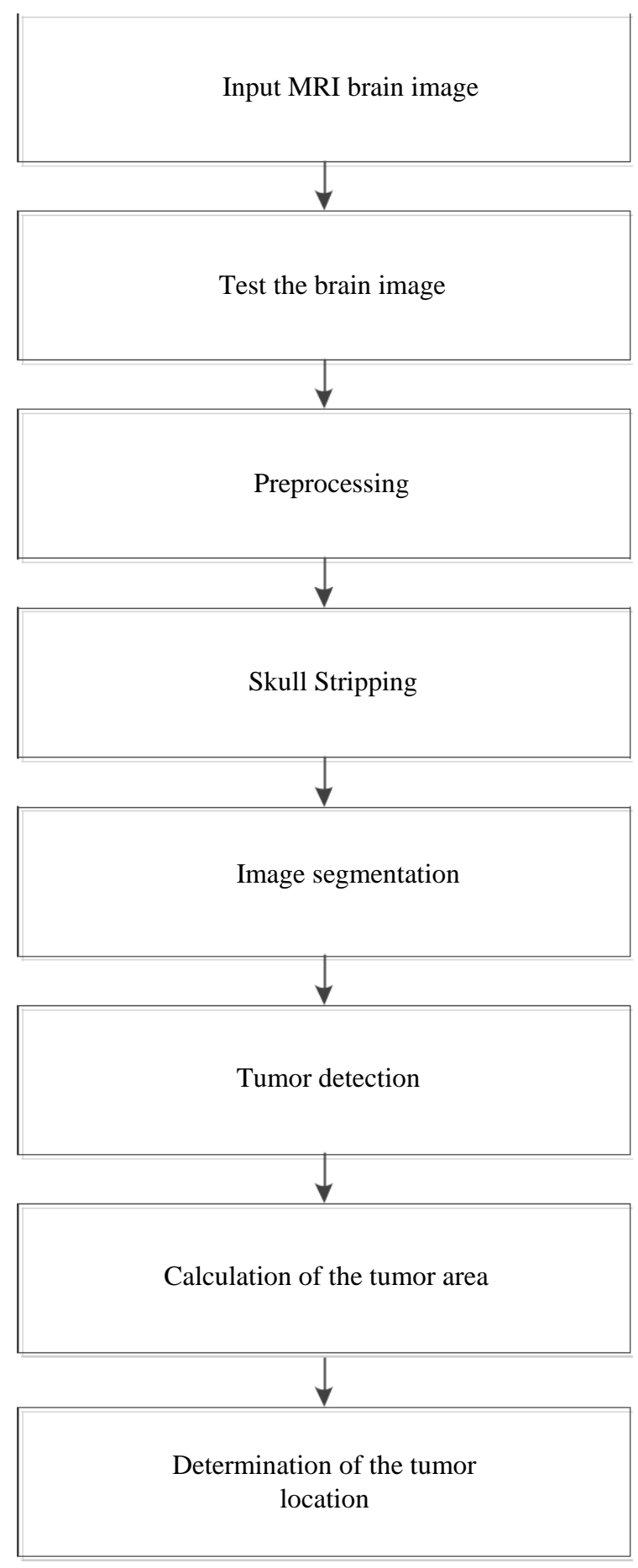

Fig - 1: Implementation procedure

\section{RESULTS}

When the input image is loaded into the test image process, the process classifies as the image into two types: the brain image with tumor and the brain image without tumor. Fig- 2 describes the input brain images and their results. 


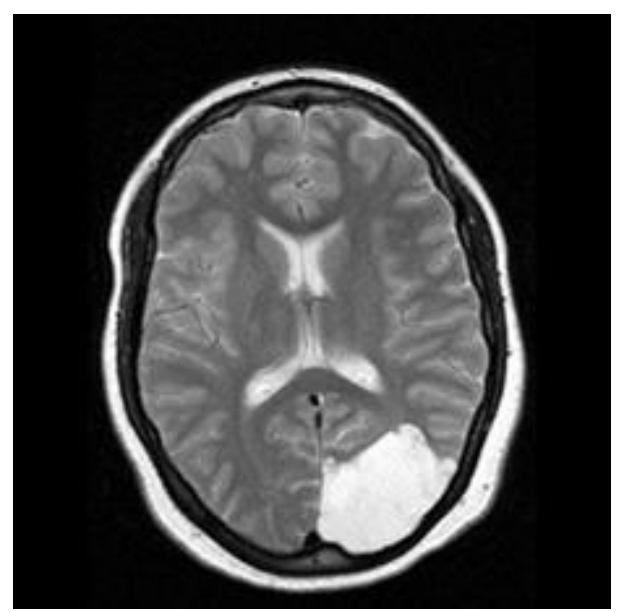

Input brain image with tumor region

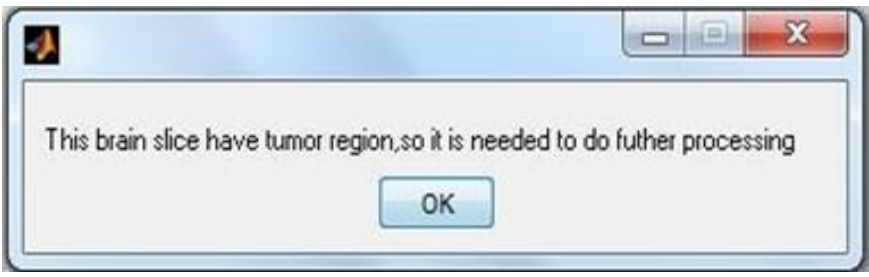

The result of the input brain image with tumor region

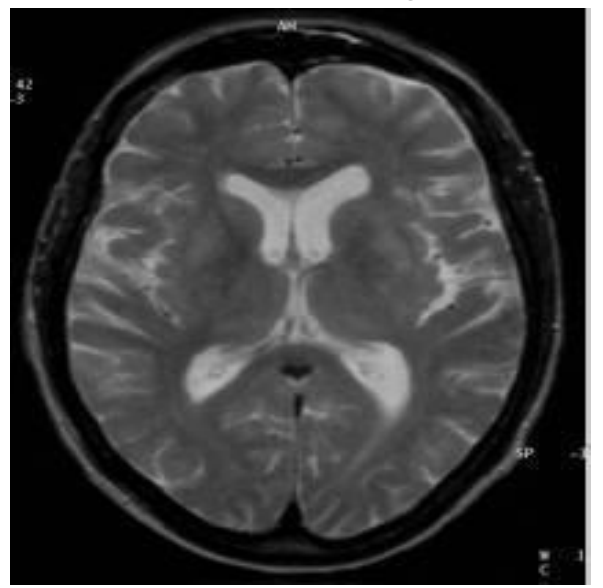

Fig - 2 : The input brain image without tumor region

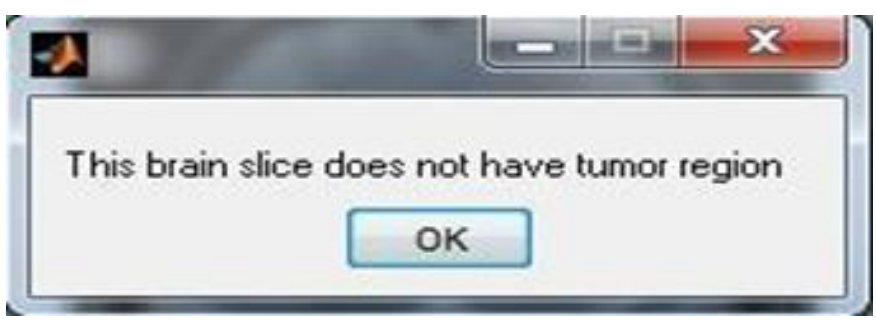

The result of the input brain image without tumor region

Fig- 3 : The input brain images and their results

If the brain image has the tumor region, this image is needed to do preprocessing. The average filter is applied the input brain image. The size of the filter is $5 \times 5$ in this research. The noise is removed to get the smoothed image. The resulted image of the preprocessing step is shown in Fig-4.

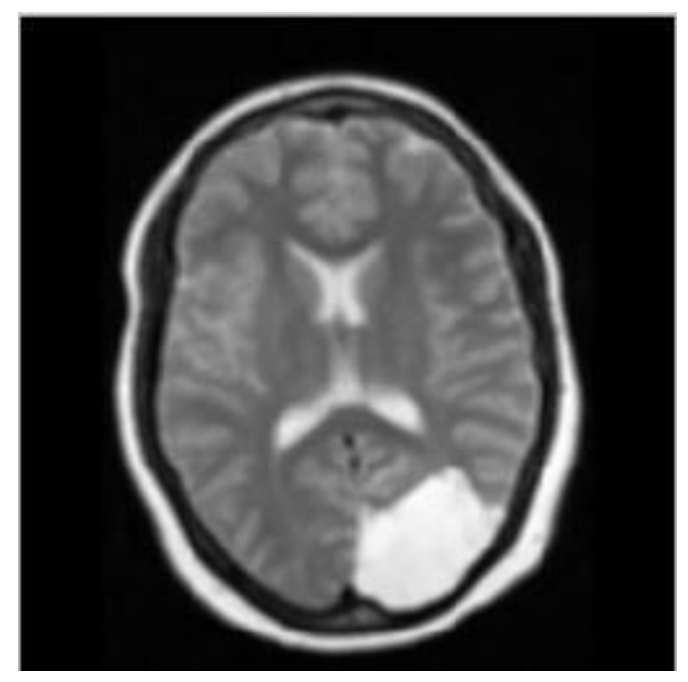

Fig- 4 : The smoothed image using average filter

After preprocessing, the resulted image is loaded into skull stripping process. Skull stripping based on threshold value can give the suitable result. Fig- 5 describes the result of the skull stripping process.

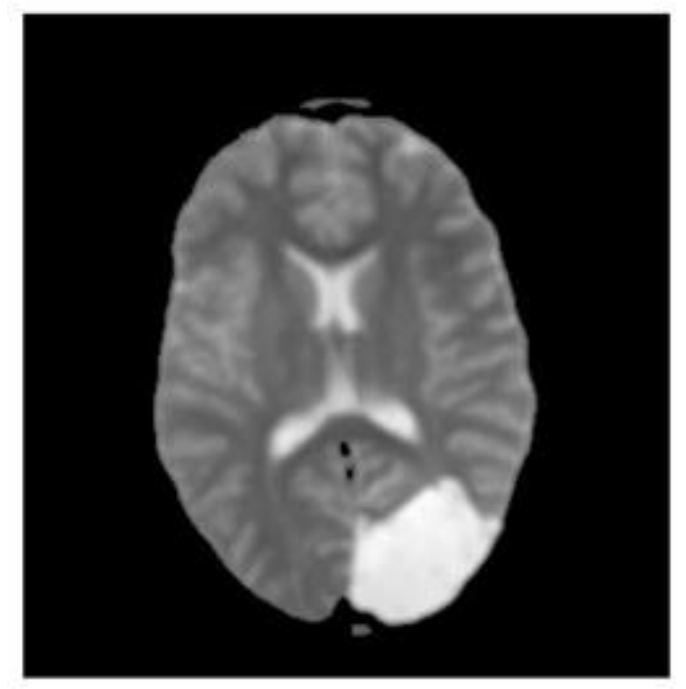

Fig- 5: The output image without skull tissues for skull stripping step

In the marker controlled watershed segmentation, the brain image without skull tissues is used to implement the step. Sobel operator is applied on the 
brain image without skull tissues and the gradient magnitude is calculated. Using the gradient magnitude, the gradient image is found out. After that, the watershed segmentation is estimated using gradient image. And then, the normal brain region and the tumor region are marked to get the final segmentation map. The segmented image obtained using marker controlled watershed segmentation is shown in Fig- 6. The image for the final segmentation map is described in Fig.-6.

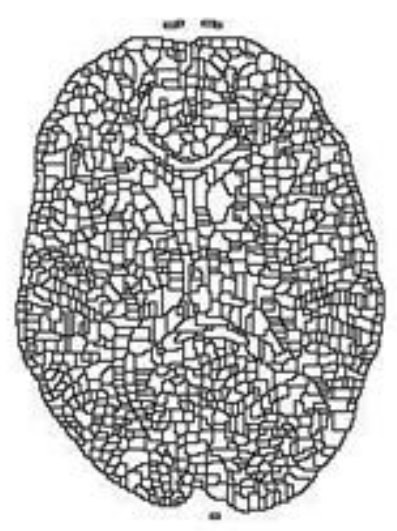

Fig- 6: The segmented image

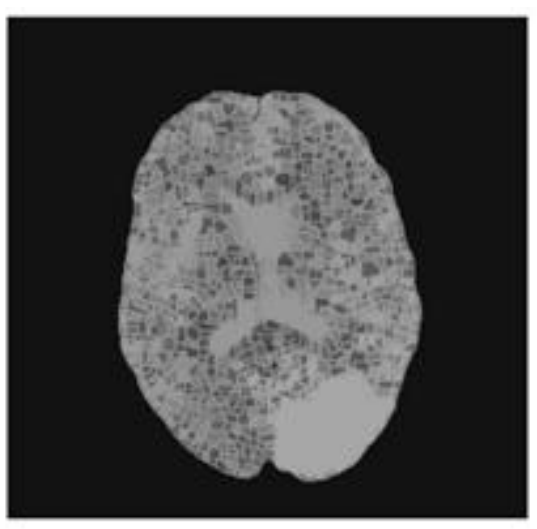

Fig.-7: The image of the tumor region for the detection process

After the tumor region had been obtained, the area of the tumor region is calculated using equation (5). The area of the tumor is get $0.3191 \mathrm{in}^{2}$. And then, the tumor location is determined using the output image obtained from the detection process. The result of the determination of the tumor location is described in Fig.-7.

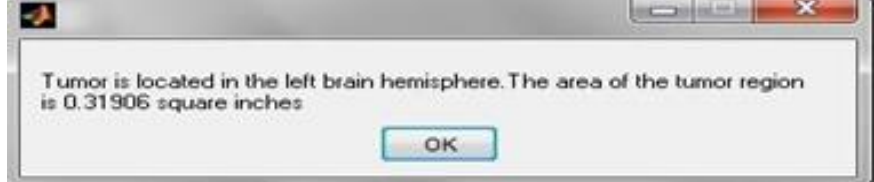

Fig- 8: The value of the tumor area and the location of the tumor

Another input brain image is shown in Fig. -9 and the result image of the tumor region is illustrated in Fig10. The area of the tumor region is $0.44301 \mathrm{in}^{2}$. Then, the location of the tumor region is shown in Fig.-11.

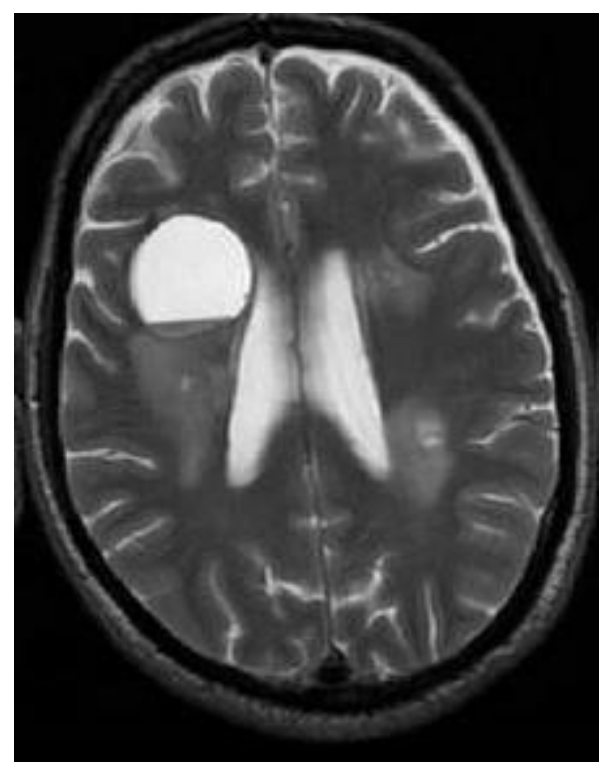

Fig.-9 : The input brain tumor image

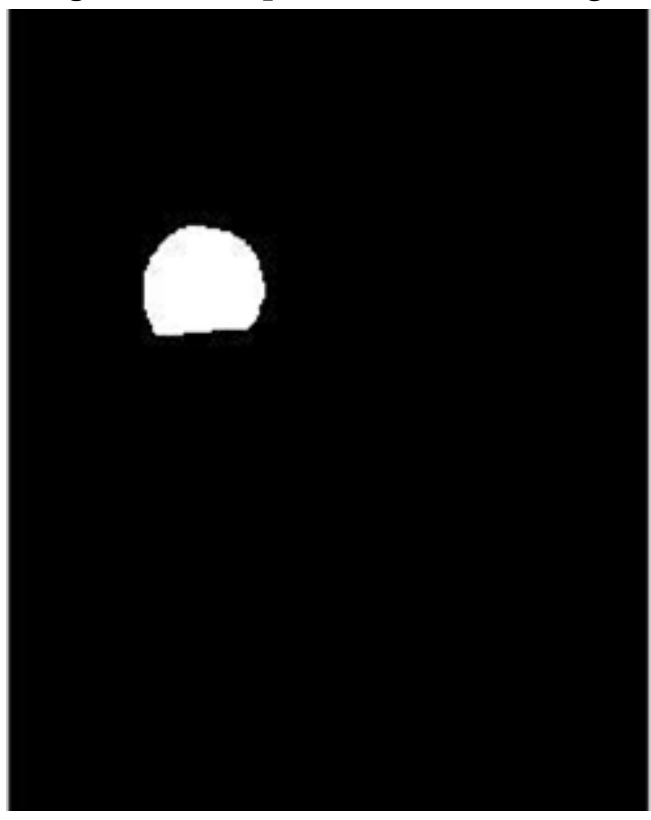

Fig.-10 : The image of the tumor region 


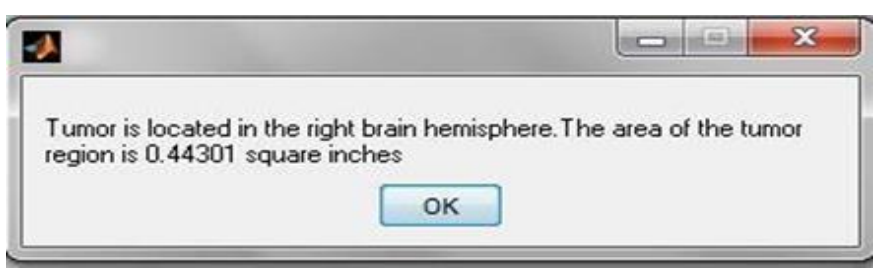

Fig-11: The value of the tumor area and the location of the tumor

\section{DISCUSSIONS}

In this paper, the brain image has been classified into normal brain image and abnormal brain image. This process can correctly classify the brain image in less execution time.

And the average filter provides the suitable smoothed image for skull stripping step. Skull stripping is needed to do only in brain image. It is not needed to do other medical image processing such as heart, lung, etc. Skull stripping based on threshold value gives the reliable result for the next processing step such as segmentation, detection, and so on. But, this skull stripping method has a drawback. When the threshold value of non-brain tissues are equal to that of the brain tissues, this skull stripping method cannot efficiently remove the skull from the brain image. Therefore the skull tissues can leave in the brain image because of this drawback. But, this drawback can be overcome using morphological operation. Then, marker controlled watershed segmentation method can efficiently segment the MRI brain tumor image. This segmentation method can reduce the problem of over segmentation and gives the suitable segmentation map for detection of the tumor. Tumor detection using morphological operation can give the exact result of the tumor region. The equation of the tumor area in this research is suitable for any shape of the tumor region. It can give the exact value of the rumor region. In this system, the location of the tumor has been determined. This method can efficiently display the location of the tumor at left hemisphere or right hemisphere.

\section{CONCLUSION}

In medical decision, the use of computer science plays an important role for analyzing various diseases. Magnetic resonance image (MRI) is a critical part in many researches. So the MRI brain image is used to implement the system. And morphological operation is used to detect the tumor region. It is easy to implement and reasonably fast. In this work, the brain image testing process has been done. This method is given the reliable result for the brain image. If the brain image has the tumor region, the further processing steps are needed to be done. The preprocessing step is important to segment the brain image. After the preprocessing, the brain image is free from noise and this smoothed image is ready to be used in further processing. In this system, a skull stripping based on threshold value has been done. This method is able to remove the skull tissues from the brain image and give the suitable result. And then, marker controlled watershed segmentation has been done. Therefore, the intensity of normal brain tissues and that of tumor region is split up into groups. After segmentation, this resulted image is divided into the normal brain region and the tumor region to get the final segmentation map. After that, the tumor region is detected from the final segmentation map using morphological operation. And the tumor region is calculated according to the equation (5). Finally, the location of the tumor region has been determined based on the pixel value of the tumor region. The right part of the image and the left part of the image are defined by manually in this process. Therefore, the location of the tumor is needed to define automatically. In this paper, the tumor region is extracted from the single MRI brain slice. Really, MRI scan take the picture of the brain in many slices. And the tumor region is not located in the single slice. Above the reason tumor volume is needed to calculate. The volume of the tumor is calculated to get the exact 
result of the tumor region. Therefore, the tumor volume is needed to calculate. The tumor volume can be calculated using Frustum model.

\section{ACKNOWLEDGMENT}

The author would like to acknowledge the head of Department of Electronic Engineering, Mandalay Technological University.

The author is highly grateful to all the teachers, Department of Electronic Engineering, Mandalay Technological University. The author specially wants to thank her supervisor Dr. Aung Soe Khaing, Department of Electronic Engineering, Mandalay Technological University for his guidance and constant encouragement throughout the work.

\section{REFERENCES}

[1]. Skull Stripping of MRI Head Scans based on Chan-Vese Active Contour Model, Online Available],

www.med.harvad.edu/AANLIB/home.html, accessed on 8 August 2013.

[2]. Magnetic Resonance Image, Online Available], www.CEwebsource.com, accessed on 18 June 2013.

[3]. Pratik P. Singhai, Siddharth A. Ladhake, "Brain Tumor DetectionusingMarkerBasedWatershed Segmentation from Digital MR images", International Journal of Innovative Technology and Exploring Engineering (IJITEE) ISSN: 2278-3075, Volume-2, Issue-5, April 2013.

[4]. A.Jeeviitha, P. Narendran, "BTS (Brain Tumor Segmentation) Based on Otus Thresholding," Indian Journal of Research, Volume:2, Issue:2, ISSN- 2250-1991, February 2013.

[5]. Manor K Kowari and Sourabh Yadav, "Brain Tumor Detection and Segmentation using Histogram Thresholding”, International Journal of Engineering and Advanced Technology (IJEAT) ISSN: 2249-898, Volume-1, Issue-4, Journal, India, April 2012.

[6]. M. Masroor Ahmed, Dzulkifli Bin Mohamad, "Segmentation of Brain MR Images for Tumor Extraction by Combining Kmeans Clustering and Perona-Malik Anisotropic Diffusion model.

[7]. Nagalkaar. V.J and Asole S.S, "Brain Tumor Detection using Digital Image Processing based on Soft Computing," Journal of Signal and Image Processing, Volume 3, Issue 3, Issn: 0976-8882, 2012.

[8]. Rajesh C.Patil, Dr. A. S.Bhalchandra, "Brain Tumor Extraction from MRI Images using MATLAB," International Journal of Electronics, Communication \& Soft Computing Science and Engineering, Volume 2, Issue 1, ISSN: 22779477.

[9]. S Jayaraman, S Esakkirajan and TVeerakumar, Digital Image Processing, 3rdEdition, Tata McGraw Hill, 2010, ISBN (13): 978-0-07014479-8, ISBN (10): 0-070114479-6.

[10]. M. C Jobin Christ, R.M.S. Paravathi, "Segmentation of Medical Image using Clustering and Watershed Algorithms", American Journal of Applied Sciences 8(2): 1349-152, 2011 ISSN 1546-9239@ 2011 Science Publication.

[11]. Dibyendu Goshal, Pinaki Pratim Acharjya, "MRI Image Segmentation using Watershed Transform", International Journal of Emerging Technology and Advanced Engineering, ISSN 2250-2459, Volume 2, Issue 4, April 2012.

[12]. "Biosignal and Biomedical Image Processing", Online Available], www.dekker.com, accessed on 11 October 2013.

[13]. Rosniza Roslan, Nursuriati Jamil and Rozi Mahmud, "Skull Stripping Magnetic Resonance Images Brain Images: region Growing versus Mathematical Morphology", International Journal of Computer Information Systems and 
Industrial Management applications, ISSN 2150-7988, Volume (2011).

\section{Cite this article as :}

V. Supraja, Kuna Haritha, Gunjalli Mounika, Chintha Manideepika, Kandikeri Sai Jeevani, "Brain Tumor Detection and Segmentation Using Watershed Segmentation and Morphological Operation", International Journal of Scientific Research in Science, Engineering and Technology (IJSRSET), Online ISSN : 2394-4099, Print ISSN : 2395-1990, Volume 8 Issue 4, pp. 304-312, July-August 2021. Available at doi : https://doi.org/10.32628/IJSRSET218451

Journal URL : https://ijsrset.com/IJSRSET218451 